\title{
Packet switch scheduler for increasing sending packet
}

\author{
Kazunori Omori, Myungryun Yoo, Takanori Yokoyama \\ Information Engineering, Tokyo City University, Tokyo, Japan \\ Email address: \\ g1381501@tcu.ac.jp (K. Omori), yoo@cs.tcu.ac.jp (M. Yoo), yokoyama@cs.tcu.ac.jp (T. Yokoyama)
}

\section{To cite this article:}

Kazunori Omori, Myungryun Yoo, Takanori Yokoyama. Packet Switch Scheduler for Increasing Sending Packet. International Journal of Intelligent Information Systems. Special Issue: Logistics Optimization Using Evolutionary Computation Techniques.

Vol. 4, No. 2-1, 2015, pp. 7-12. doi: 10.11648/j.ijiis.s.2015040201.12

\begin{abstract}
Recently, the need of the high speed packet switch is increased. The Re-2DRR scheduling algorithm based on 2DRR scheduling algorithm provides high throughput communication on a packet switch. However, computer network is using many cases, that huge data communication, complex, and other. This paper proposes a new method to increase choices in algorithm variation for three specific systems and more easily implementation than Re-2DRR. The effectiveness of the proposed algorithm is shown through simulation studies.
\end{abstract}

Keywords: Packet Switch Scheduling algorithm; 2DRR; High-Throughput

\section{Introduction}

In recent years, the number of traffic packet network is on the increase [1], [2]. And they are making many kinds of systems on a computer networks, and they use to a computer network for special use for example, multimedia communications [3], [4], and other. They are needed more the high speed packet switch scheduler for special use [5]. Richard presented the basic two Dimensional Round Robin (2DRR) scheduling algorithm [6]. The four matrixes, Request Matrix (RM), Pattern Matrix (PM), Scheduling Matrix (SM), and Allocation Matrix (AM) are used in the basic 2DRR. Each matrix size is $\mathrm{N} x \mathrm{~N}$, where $\mathrm{N}$ means the number of inputs and outputs [7]. The basic 2DRR provides high throughput, fair access [8], [9] and simple working on a packet switch. However, few input and output node could not be permissioned in some timeslots because the basic 2DRR use only value of PM to running scheduling. It occurs decreasing throughput and some transmission delay.

The Repetitive two Dimensional Round Robin (Re-2DRR) scheduling algorithm provides higher throughput than that of the basic 2DRR scheduling algorithm [10]. The Re-2DRR is using new matrix about Sub-AM. It is solve the 2DRR delay point. Although the Re-2DRR do making Sub-AM many times, and it hindered an implementation.

In this paper, we propose two method based on Re-2DRR. And we increase choices in algorithm for special use. First method is changing to making Sub-AM operation that the basic Re-2DRR was remaking many times at it have empty packets. If this method recognize an empty packet at AM, that just made Sub-AM group once time. It is suppression a repeat operation. Second method is making priority port in Re-2DRR. It enables high throughput at specific port.

\section{Related Works}

\subsection{The Basic Two Dimensional Round Robin (2DRR)}

In an $N$ x $N$ switch (Fig.1), up to $N$ different requests can be simultaneously served by the switch in one time slot such that no two requests are in the same row or column in the request matrix. In order to select such $N$ elements of the request matrix, $\mathrm{RM}$, the basic 2DDR examines elements of RM that belong to generalized diagonals.

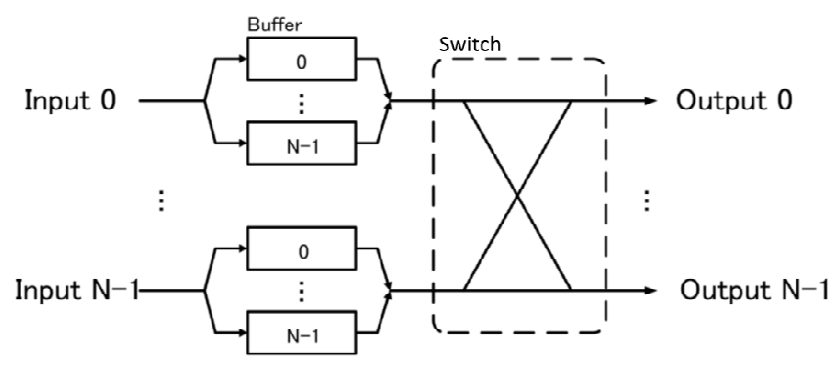

Figure 1. NxN Switch

Definition 1: A generalized diagonal is a set of $N$ elements in an $N \times N$ matrix, such that no two elements are in the same row or column. 
There are $N$ ! different generalized diagonals in an $N \times N$ matrix. The basic 2DRR algorithm uses only $N$ of these diagonals by selecting one basic diagonal and then generating the remaining $N-1$ ones by shifting the basic diagonal across the matrix (so that each matrix element is covered by one of the $N$ diagonals). That is, by sweeping a generalized diagonal pattern of length $N$ through the request matrix, all $N^{2}$ input output pairs in the request matrix can be satisfied in $N$ time slots. This property is used to guarantee a minimum amount of service to each input/output queue.

The basic 2DRR scheduling algorithm operates in repeating cycles of $N$ time slots in which the time slots of each cycle are indexed by the variable $L$, which takes on values from 0 through $N-1$. The following 4 matrices are assumed.

1) Request Matrix (RM)

Each entry $R M[R, C]$ is binary with the semantics:

$\mathrm{RM}[\mathrm{R}, \mathrm{C}]=1$, if there is at least one request for a connection from output $\mathrm{R}$ to output $\mathrm{C}$

0 , otherwise

2) Scheduling Matrix (SM)

Each entry $\mathrm{SM}[\mathrm{R}, \mathrm{C}]$ contains an integer between 0 and $\mathrm{N}-1$ inclusive where

$$
S M[R, C]=(C-R) \bmod N
$$

If $\mathrm{SM}[\mathrm{R}, \mathrm{C}]=\mathrm{K}$, then $\mathrm{RM}[\mathrm{R}, \mathrm{C}]$ is covered by diagonal pattern K.

3) Pattern Sequence Matrix (PM)

Each entry $\mathrm{PM}[\mathrm{I}, \mathrm{J}]$ is an integer between 0 and $\mathrm{N}-1$ inclusive with the semantics:

$\mathrm{PM}[\mathrm{I}, \mathrm{J}]=\mathrm{K}$ implies that when the timeslot index $\mathrm{L}$ of a cycle is equal to $\mathrm{J}$, then the I-th diagonal pattern in the sequence applied by the algorithm is the one numbered $\mathrm{K}$ in the diagonal pattern matrix. The ordering index I varies from 0 to $\mathrm{N}-1$.

4) Allocation Matrix (AM)

It is binary entries and the semantics:

$$
A M[R, C]=\left\{\begin{array}{cc}
1, & \text { if a connection is allocated } \\
0, & \text { from input } R \text { to output } C
\end{array}\right.
$$

At the beginning of time slot $L$ in a cycle, all entries of the allocation matrix are set to zero. Then a sequence of $N$ diagonal patterns is applied to the request matrix in the order specified by the pattern sequence matrix PM. That is, the diagonal pattern with index $P M[0, L]$ is applied first followed by diagonal pattern $P M[1, L]$. . P $P M[N-1, L]$. As these diagonal patterns are overlaid on the request matrix, the entry $A M[R, C]$ is set to 1 at the $I$-th point in the sequence if the following conditions are true.

- $R M[R, C]=1$

- Input $R$ and output $C$ are still available for allocation (i.e., they have not been allocated to a different connection by a previously applied diagonal in the current time slot)
- $S M[R, C]=K$. where $P M[l, L]=K$

The above scheduling procedure is repeated for each cycle of $N$ successive time slots. That is after a cycle has been completed with the use of column $N-1$ of the pattern sequence matrix.

The basic 2DRR algorithm provides a fairness guarantee that each of the $N^{2}$ input/output queues will receive at least one opportunity for service during every cycle of $N$ time slots. However, few input and output node could not be permissioned in some timeslots because the basic 2DRR use only value of PM to running scheduling. It occur scheduler tightly and throughput delay.

\subsection{The Repetitive two Dimensional Round Robin $(\operatorname{Re}-2 D R R)$}

Re-2DRR based on the basic 2DRR. The Re-2DRR creates Sub-AM if AM is not usable because of empty sending packet. If Sub-AM is not better than AM, scheduling algorithm retry creating Sub-AM by another SM, but that retrying have a limit All created Sub-AM is not better than AM, the Re-2DRR chooses either AM or Sub-AM.

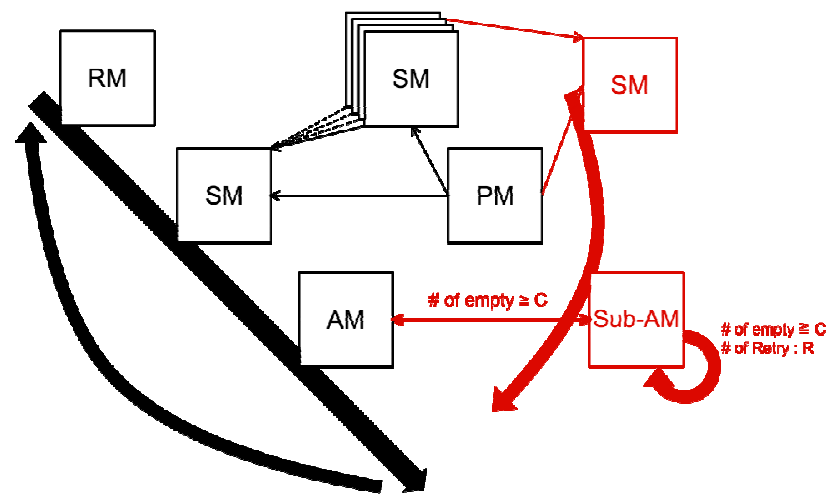

Figure 2. Re-2DRR Scheduling Algorithm

Fig. 2 is Re-2DRR Scheduling Algorithms figure. The black color part shown the basic 2DRR scheduling work, and the red color part is the Re-2DRR scheduling works.

In the Re-2DRR scheduling algorithm, two thresholds are used. The first threshold is the number of empty sending packet for decision of available AM (threshold "C") and the second threshold is the upper limit of retrying to create Sub-AM (threshold "R"). The scheduler works more effectively by setting two thresholds optionally.

The Re-2DRR can operation flexibly by changing that two threshold. In threshold $\mathrm{C}$, it changing probability of operating Re-2DRR. And threshold $\mathrm{R}$ is changing probability of algorithm chose Sub-AM and number of making Sub-AMs. In table 1 show a variation of the Re-2DRR operation results by $\mathrm{N}=4$.

We recognize the operation of making Sub-AM is burdened with implementation. And the Re-2DRR is making for high throughput at all port in packet switch communications. 
Table 1. Result of Re-2DRR in simulation ( $N=4)$

\begin{tabular}{llllll}
\hline C & R & Making Sub-AM & Sending by Sub-AM & End Time & Ave Packet Wait Time \\
\hline & 1 & 18833 & 4315 & 277192 & 138556 \\
1 & 2 & 33922 & 5494 & 277004 & 138433 \\
& 3 & 49911 & 6227 & 277325 & 138635 \\
& 4 & 61212 & 6655 & 276782 & 138430 \\
& 1 & 8465 & 1159 & 277082 & 138483 \\
2 & 15763 & 1472 & 277196 & 138536 \\
& 2 & 23608 & 1527 & 277161 & 138560 \\
& 3 & 29519 & 1593 & 277045 & 138485 \\
& 4 & 4042 & 257 & 277077 & 138554 \\
& 1 & 8284 & 334 & 277278 & 138651 \\
& 2 & 12703 & 363 & 277351 & 138692 \\
\end{tabular}

\section{System to Assume}

In this paper we define three specific systems. It is make more packet loading.

\subsection{Less than N Port Have More Packet Load}

It is less than $N$ port have more packet loading. It like Fig. 3 (a). In Fig.3 (a) mean a communication of Input 1 to Output 1 (point of red) use more packet traffics.

This traffic is estimated to be about some node use big data communication temporary.

\subsection{Static N Port Have More Packet Load}

This specific system is have more packet load at number of $N$ communications. Example in Fig.3 (b) mean static number of 4 input to number of 4 output (place of red) have more packet loading.

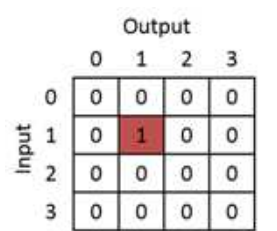

(a)

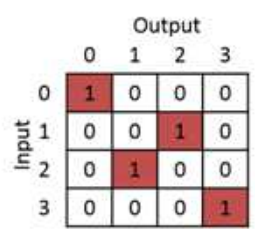

(b)

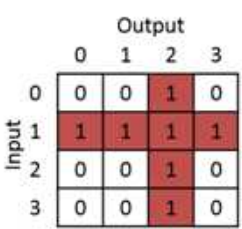

(c)
Figure 3. A place of more packet load

This traffic is estimated to be about number of $N$ nodes and number of $N$ nodes make static communication and use a big data.

\subsection{One to All and All to One Port Have More Packet Load}

This specific system is like a broadcast communication. In Fig.3 (c) mean input 1 to all output, and all input to output 2 have more packet load.

\section{Improvement}

In this paper, we improve portion of Re-2DRR. And we regarded two methods.

\subsection{Selectable Sub-AM Group}

In Re-2DRR, if Sub-AM worse than AM about the number of empty packets, scheduler make more another one Sub-AM for $\mathrm{R}$ times. This method improve about portion of making Sub-AM. If AM have many empty packet, this algorithm making the number of G Sub-AMs. After that, scheduler checking the better in Sub-AM group and select the best Sub-AM. If AM is better than Sub-AM groups Sub-AMs after made Sub-AM group, that algorithm send by AM.

In this method suppression a repeat making Sub-AM operation.

In Fig.4, the black color is same of Re-2DRR. The red color is this method point.

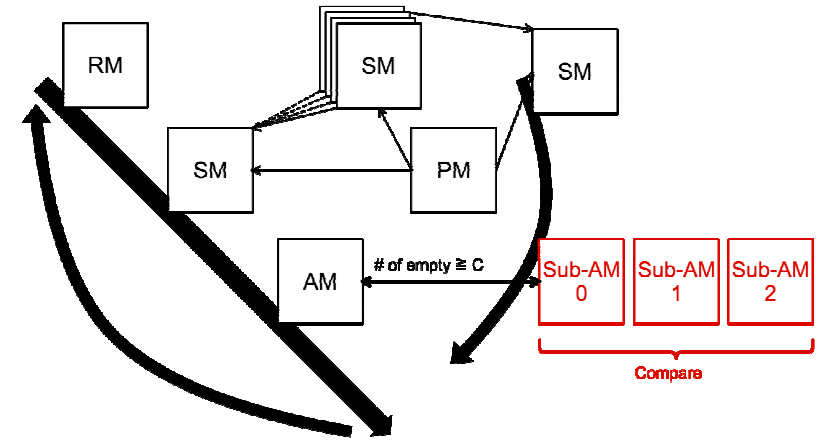

Figure 4. Selectable Sub-AM group

\subsection{Make Priority Port}

The basic Re-2DRR is choose AM or Sub-AM about the number of empty packet. This method is making priority port in operation of checking and choosing SM or Sub-SM of the basic Re-2DRR.

This method algorithm is choosing AM or Sub-AM preferentially about a set priority. For example in Fig.5, it is example of AM or Sub-AM. And place of yellow are priority ports in this time. This method algorithm choosing (b), because place of yellow is priority ports have permission of sending packet. So this method algorithm don't need the threshold of C, because this method just judge by priority port. 


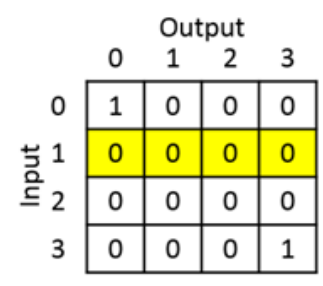

(a)

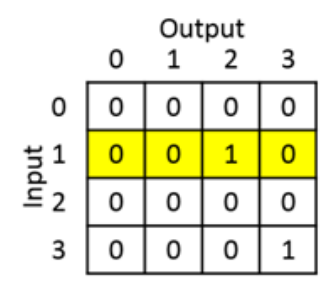

(b)
Figure 5. Example of priority port

\section{Validation}

To validate proposed two methods on Re-2DRR, several numerical tests are performed. We compared proposed selectable Sub-AM group on Re-2DRR, setting priority port on Re-2DRR and basic Re-2DRR. Numerical tests are performed with $N \times N$ matrix $(N=4)$. The throughput and the finish time of all sending packets are compared. The throughput is average waiting time of all packets in packet switch buffer. $C$ and $R$ is the threshold in Re-2DRR. $C$ is the number of empty sending packet for decision of available $\mathrm{AM}$ and $R$ is the upper limit of retrying to create Sub-AM. $G$ is the threshold in the method of selectable Sub-AM group about the number of Sub-AM in Sub-AM group. The method of making priority port isn't need $C$.

Table 2, 3, 4, 5, 6, 7 and 8 show the comparison of results by basic Re-2DRR and two method on Re-2DRR. Table 2 is the results on more treble packet load to $(1,1)$ port. Table 3 , 4 and 5 are the results on the number of $N$ port (in this time $\mathrm{N}$ is 4 : $(0,0),(1,1),(2,3)$ and $(3,2)$ ports) have more treble packet load. Table 4 is the results about each four ports average of packet waiting time. And table 5 is the results about four ports maximum of packet waiting time. Table 6,7 and 8 are the results on input 1 to all output and all input to output 2 have more treble packet load. Table 7 is the results about each seven ports average of packet waiting time. And table 4 is the results about seven ports maximum of packet waiting time.

In table 2, Sub-AM group $(C: 1 G: 4)$ and Sub-AM group ( $C: 1 \quad G: 3)$ results close to Re-2DRR $(C: 1 \quad R: 4)$ and Re-2DRR( $C: 1 R: 3)$ results except for Make Sub-AM group. The basic Re-2DRR don't making Sub-AM groups, so need extravagant making Sub-AM operation. However, method of Sub-AM group on Re-2DRR made Sub-AM group in advance. It mean the method of Sub-AM group in Re-2DRR can working by less making Sub-AM operation. The $(1,1)$ ports throughput of method of making priority port on Re-2DRR is more desirable than those of the others. But another values are increasing. But Priority $(R: 3)$ keep values increasing and keep high $(1,1)$ ports throughput.

In table 3 , the method of making Sub-AM group on Re-2DRRs results are close to the basic Re-2DRR. And it shows the method of making Sub-AM groups making Sub-AM operation are less than the basic Re-2DRRs making Sub-AM operation. However the method of making priority port on Re-2DRR is not have enough effects. This method can't cause empty packet sending, because it give very high priority to setting four priority ports at $(0,0),(1,1),(2,3)$ and $(3,2)$. There are get big priority area, so that method increasing chose AM. In table 6, 7 and 8, this method setting seven priority port at same of more packet load ports, which is get more big priority area. It is block to making Sub-AM. Priority $(R: 4)$ limit in table 6,7 and 8 is setting priority at input 1 about $(1,0),(1,1),(1,2)$ and $(1,3)$. It mean setting priority area narrowing. In conclusion, the method of making priority port on Re-2DR R have a just effect on one priority port like table 2 .

Table 2. Comparison on $(1,1)$ port have more packet load

\begin{tabular}{llllllll}
\hline & $\begin{array}{l}\text { Make } \\
\text { Sub-AM }\end{array}$ & $\begin{array}{l}\text { Send by } \\
\text { Sub-AM }\end{array}$ & End Time & Ave Wait Time & $\begin{array}{l}\text { Make } \\
\text { Sub-AM group }\end{array}$ & $\begin{array}{l}\text { (1, 1) } \\
\text { Ave Wait }\end{array}$ & $\begin{array}{l}\text { (1, 1) } \\
\text { MAX Wait Time }\end{array}$ \\
\hline Re-2DRR $(C: 1 \quad R: 4)$ & 543976 & 6908 & 398402 & 1561.236 & - & 2667 & 4456 \\
Re-2DRR $(C: 1 R: 3)$ & 414737 & 6042 & 399563 & 1564.182 & - & 2677 & 4516 \\
Sub-AM group $(C: 1 G: 4)$ & 546784 & 6077 & 394756 & 1553.451 & 136696 & 2643 & 4735 \\
Sub-AM group $(C: 1 G: 3)$ & 418731 & 5916 & 397935 & 1560.104 & 139577 & 2662 & 4541 \\
Priority $(R: 4)$ & 1134177 & 136500 & 396138 & 1670.850 & - & 1106 & 2876 \\
Priority $(R: 3)$ & 583186 & 115229 & 397203 & 1659.550 & - & 1560 & 4093 \\
\hline
\end{tabular}

Table 3. Comparison on the number of $N$ port have more packet load

\begin{tabular}{|c|c|c|c|c|c|}
\hline & Make Sub-AM & Send by Sub-AM & End Time & Ave Wait Time & Make Sub-AM group \\
\hline Re-2DRR ( $C: 1 R: 4)$ & 332910 & 67320 & 423527 & 1993.703 & - \\
\hline $\operatorname{Re}-2 \mathrm{DRR}(C: 1 R: 3)$ & 257648 & 55580 & 424641 & 1995.891 & - \\
\hline Sub-AM group ( $C: 1 G: 4)$ & 386220 & 39035 & 423077 & 1989.144 & 96555 \\
\hline Sub-AM group $(C: 1 G: 3)$ & 285531 & 36749 & 425153 & 1998.776 & 139577 \\
\hline Priority $(R: 4)$ & 90322 & 73489 & 440118 & 2047.618 & - \\
\hline Priority $(R: 3)$ & 90400 & 73511 & 445007 & 2065.108 & - \\
\hline
\end{tabular}


Table 4. Comparison on the number of N ports have more packet loads average of packet waiting time

\begin{tabular}{|c|c|c|c|c|}
\hline & $(0,0)$ & $(1,1)$ & $(2,3)$ & $(3,2)$ \\
\hline Re-2DRR ( $C: 1 R: 4)$ & 2649 & 2659 & 2668 & 2661 \\
\hline $\operatorname{Re}-2 \mathrm{DRR}(C: 1 R: 3)$ & 2656 & 2676 & 2647 & 2654 \\
\hline Sub-AM group ( $C: 1 G: 4)$ & 2653 & 2659 & 2670 & 2653 \\
\hline Sub-AM group ( $C: 1 G: 3)$ & 2661 & 2692 & 2687 & 2647 \\
\hline Priority $(R: 4)$ & 2403 & 2589 & 2767 & 2582 \\
\hline Priority $(R: 3)$ & 2466 & 2593 & 2780 & 2612 \\
\hline
\end{tabular}

Table 5. Comparison on the number of $N$ ports have more packet loads maximum of packet waiting time

\begin{tabular}{lllrr}
\hline & $\mathbf{( 0 , 0 )}$ & $\mathbf{( 1 , \mathbf { 1 } )}$ & $\mathbf{( 2 , \mathbf { 3 } )}$ & $\mathbf{( 3 , \mathbf { 2 } )}$ \\
\hline Re-2DRR $(C: 1 R: 4)$ & 4794 & 4646 & 4628 & 4620 \\
Re-2DRR $(C: 1 R: 3)$ & 4480 & 4739 & 4519 & 4660 \\
Sub-AM group $(C: 1 G: 4)$ & 4663 & 4651 & 4597 & 4468 \\
Sub-AM group $(C: 1 G: 3)$ & 4940 & 4773 & 4547 & 4713 \\
Priority $(R: 4)$ & 4813 & 4954 & 4574 \\
Priority $(R: 3)$ & 4783 & 4636 & 4877 \\
\hline
\end{tabular}

Table 6. Comparison on input 1 to all output and all input to output 2 have more packet load

\begin{tabular}{lllll}
\hline & Make Sub-AM & Send by Sub-AM & End Time & Ave Wait Time \\
\hline Re-2DRR $(C: 1 R: 4)$ & 1857306 & 99209 & 796028 & 2991.344 \\
Re-2DRR $(C: 1 R: 3)$ & 1430890 & 95902 & 799003 & 2998.931 \\
Sub-AM group $(C: 1 G: 4)$ & 2162800 & 96774 & 802024 & 3007.809 \\
Sub-AM group $(C: 1 G: 3)$ & 1640709 & 95880 & 807698 & 3024.896 \\
Priority $(R: 4)$ & 0 & 0 & 801634 & 3122.378 \\
Priority $(R: 3)$ & 0 & 0 & 806794 & 3133.948 \\
Priority $(R: 4)$ limit & 73350 & 3689 & 799421 & 3103.385 \\
\hline
\end{tabular}

Table 7. Comparison on input 1 to all output and all input to output 2 have more packet load s average of packet waiting time

\begin{tabular}{|c|c|c|c|c|c|c|c|}
\hline & $(1,0)$ & $(1,1)$ & $(1,2)$ & $(1,3)$ & $(0,3)$ & $(2,2)$ & $(3,2)$ \\
\hline Re-2DRR ( $C: 1 R: 4)$ & 3851 & 3946 & 3894 & 3797 & 3836 & 3900 & 3880 \\
\hline Re-2DRR ( $C: 1 R: 3)$ & 3552 & 3653 & 5250 & 3171 & 3333 & 3339 & 3740 \\
\hline Sub-AM group ( $C: 1 G: 4)$ & 3352 & 3550 & 5264 & 3557 & 3706 & 3618 & 3169 \\
\hline Sub-AM group ( $C: 1 G: 3)$ & 3519 & 3691 & 5320 & 3280 & 3376 & 3433 & 3744 \\
\hline Priority $(R: 4)$ & 3910 & 3860 & 3905 & 3892 & 3908 & 3944 & 3954 \\
\hline Priority ( $R: 3)$ & 3859 & 3861 & 3951 & 3961 & 3973 & 3968 & 3901 \\
\hline Priority $(R: 4)$ limit & 3803 & 3899 & 3892 & 3909 & 3910 & 3955 & 3844 \\
\hline
\end{tabular}

Table 8. Comparison on input 1 to all output and all input to output 2 have more packet loads maximum of packet waiting time

\begin{tabular}{|c|c|c|c|c|c|c|c|}
\hline & $(1,0)$ & $(1,1)$ & $(1,2)$ & $(1,3)$ & $(0,3)$ & $(2,2)$ & $(3,2)$ \\
\hline Re-2DRR ( $C: 1 R: 4)$ & 9169 & 9423 & 9351 & 9460 & 8808 & 8458 & 8395 \\
\hline $\operatorname{Re}-2 \mathrm{DRR}(C: 1 R: 3)$ & 8111 & 8143 & 8892 & 7378 & 7935 & 8032 & 8462 \\
\hline Sub-AM group ( $C: 1 G: 4)$ & 8185 & 7961 & 9095 & 8018 & 7906 & 8011 & 7502 \\
\hline Sub-AM group ( $C: 1 G: 3)$ & 8018 & 8424 & 9499 & 7938 & 8008 & 8400 & 8808 \\
\hline Priority $(R: 4)$ & 8451 & 8753 & 9262 & 8659 & 9320 & 9339 & 9033 \\
\hline Priority $(R: 3)$ & 8549 & 8936 & 9376 & 9054 & 8881 & 9117 & 8881 \\
\hline Priority $(R: 4)$ limit & 8520 & 8728 & 8915 & 8648 & 8712 & 8934 & 8678 \\
\hline
\end{tabular}




\section{Conclusion}

A new two method on Re-2DRR is proposed in this paper. The proposed method of making Sub-AM group on Re-2DRR is designed for less making Sub-AM operation. It mean more easer working and implementation. And the method of making priority port on Re-2DRR is designed for the high throughput in one specific priority port communications.

\section{Acknowledgements}

This work is supported in part by JSPS KAKENHI Grant Number 24500046.

\section{References}

[1] M. Takajo, S. Kimura and Y. Ebihara, "A Proposal of Grouped Two-Dimensional Round Robin Schedulers for Multiaccess Communications," The Transactions of the Institute of Electronics, Information and Communication Engineers B, Vol. J82-B, No. 4, 1999, pp. 560-568.

[2] Craig Partridge et al., “A 50-Gb/s IP Router," IEEE/ACM Transactions of Networking, Vol. 6, No. 3, 1998, pp. 237-248.
[3] T. Tsuda, "Multimedia Communication System," The Institute of Image Information and Television Engineers, Vol. 45, No. 1, 1991, pp. 31-34.

[4] K. Nishimura, T. Mori and Y. Ishibashi, "Video-on-Demand with Multiple Readouts," The Institute of Image Information and Television Engineers, Vol.48, No. 3, 1994, pp. 287-294.

[5] ITU-T: ITU-T Recommendation I.371, 1993, pp.2-4.

[6] R. O. LaMaire, "Two-Dimensional Round-Robin Schedulers for Packet Switches with Multiple Input Queues," IEEE/ACM Transactions on Networking, Vol. 2, No. 5, 1994, pp. 471-482.

[7] M. J .Karol, M. G. Hluchyj and S. P. Morgan, "Input versus output queueing on a space-division packet switch," IEEE Trans. Commun., Vol. COM-35, 1987, pp. 1347-1356.

[8] J. Wong, J.p. Sauve, and J.S. Field, "A Study of Fairness in Packet Switching Networks," IEEE Trans. Commun., col.COM-30, No.2, 1982, pp. 346-353.

[9] E. L. Hahne, "Round-Robin Scheduling for Mac-Min Fairness in Data Networks," IEEE Journal on Selected Areas in Communications, Vol. 9, No. 7, 1991, pp. 1024-1039.

[10] K. Omori, M. Yoo, T. Yokoyama, "A Packet Switch Scheduler based on 2DRR for High-Throughput," The 75th National Convention of IPSJ, 1L-4, 2013, pp. 205-206. 